\title{
ANÁLISE DO ESTILO DE VIDA ASSOCIADA AO USO DE ANTIDEPRESSIVO EM ESTUDANTES DE MEDICINA
}

\author{
Cynthia Kallás Bachur*1; Bianca Dias Rodrigues²; Jessica Lara Lazzari Ducas²; Julia Eid de \\ Mello²; Laurynês de Castro $^{2}$; Márcia Simei Zanovello Duarte ${ }^{3}$ and Sarah da Silva Candido ${ }^{4}$
}

${ }^{1}$ Fisioterapeuta. Docente do Curso de Fisioterapia e de Medicina da Universidade de Franca -Franca - S.P. -

Brasil; '2Estudantes do Curso de Medicina da Universidade de Franca (UNIFRAN), Franca - SP - Brazil;

${ }^{3}$ Psicóloga. Mestre. Docente do Curso de Medicina da Universidade de Franca -Franca - S.P. - Brasil;

${ }_{4}^{4}$ Fisioterapeuta. Docente do Curso de Fisioterapia da Universidade de Franca -Franca - S.P. - Brasil

\begin{abstract}
ARTICLE INFO
Article History:

Received $16^{\text {th }}$ February, 2021

Received in revised form

$11^{\text {th }}$ March, 2021

Accepted $21^{\text {st }}$ April, 2021

Published online $30^{\text {th }}$ May, 2021

\section{Key Words:}

Antidepressivos; Estilo de Vida; Estudantes; Distúrbios Psiquiátricos.

*Corresponding author:

Cynthia Kallás Bachur

RESUMO

Objetivo: Avaliar o estilo de vida a partir do instrumento FANTÁSTICO e identificar o uso de medicamentos antidepressivos associados a determinados comportamentos, em estudantes de medicina. Métodos: Estudo de caráter descritivo e transversal, de base populacional e amostra por conveniência. Realizado com a participação dos estudantes da Faculdade de Medicina privada de Franca, que aceitaram responder o questionário de forma online (Google Forms). Utilizou-se o questionário sócio demográfico e o FANTASTICO, que possui 25 questões fechadas explorando 9 domínios sobre os componentes físicos, psicológicos e sociais do estilo de vida. Resultados: Participaram 105 estudantes,destes, 82 mulheres e 23 homens com idade média de 22,62 $\pm 2,81$ anos,sendo $19(19,9 \%)$ do $1^{\circ}$ ano, $23(24,1 \%)$ do $2^{\circ}$ ano, $16(16,8 \%)$ do $3^{\circ}$ ano e $47(49,3 \%)$ do $4^{\circ}$ ano. A classificação geral foi "Bom", nenhum participante apresenta pontuação na categoria "Necessita Melhorar", 2 (1,9\%) se enquadram na categoria "Excelente". Os domínios que mais necessitam de mudança são "Tipo de Comportamento" (1,3 pontos), "Nutrição" (1,5 pontos) e "Atividade" (1,6 pontos). Conclusão: Os resultados do estudo sugerem que o uso de antidepressivos foi fator preditor para uma indicação de Estilo de Vida não saudável de acordo com o questionário Estilo de Vida FANTÁSTICO.
\end{abstract}

Copyright (C) 2021, Bernardo Amarante de Lara and Silvana Alba Scortegagna. This is an open access article distributed under the Creative Commons Attribution License, which permits unrestricted use, distribution, and reproduction in any medium, provided the original work is properly cited.

Citation: Cynthia Kallás Bachur; Bianca Dias Rodrigues; Jessica Lara Lazzari Ducas; Julia Eid de Mello; Laurynês de Castro; Márcia Simei Zanovello Duarte and Sarah da Silva Candido, 2021. "Análise do estilo de vida associada ao uso de antidepressivo em estudantes de medicina", International Journal of Development Research, 11, (05), 47313-47317.

\section{INTRODUÇÃO}

A depressão é definida como um transtorno de humor crônico e é proveniente de mecanismos patogênicos relacionados à diminuição de monoaminas, que são principalmente a dopamina, serotonina e norepinefrina ${ }^{(1)}$. Caracteriza-se por sintomas de tristeza, desânimo, perda de apetite, dificuldade de concentração e sentimentos de culpa. Devido a essa sintomatologia, o transtorno gera grandes mudanças na qualidade de vida do paciente e da família ${ }^{(2)}$. A nível mundial, foi estimado pela Organização Mundial da Saúde (OMS), em 2015, mais de 300 milhões de pessoas com depressão, o que corresponde a 4,4\% da população mundial, e um número muito próximo sofrendo de ansiedade. Além disso, a depressão foi classificada como maior colaboradora da incapacidade produtiva ${ }^{(3)}$. Em relação ao Brasil, dados do Ministério da Saúde mostram que cerca de 23 milhões de pessoas, $12 \%$ da população, precisam de atendimento em saúde mental, entretanto menos da metade dos portadores de transtornos mentais buscam tratamento nos serviços de saúde ${ }^{(4)}$.
Dois tipos de abordagens terapêuticas podem ser utilizados para a depressão: a farmacológica e a não farmacológica. Nesta última destaca-se a psicoterapia, fototerapia e eletroconvulsoterapia; o tratamento farmacológico é feito a partir do uso de fármacos antidepressivos ${ }^{(5)}$. Os medicamentos podem ser subdivididos nas seguintes classes: antidepressivos tricíclicos (ADTs), inibidores seletivos da recaptação da serotonina (ISRSs), inibidores seletivos da recaptação da serotonina e noradrenalina (ISRSNs), inibidores da monoaminooxidase (IMAOs) e os antidepressivos atípicos. Atualmente, os ISRSNs são os mais utilizados na população jovem, já que apresenta menores efeitos colaterais. Destaca-se crescente aumento na prescrição de antidepressivos nos últimos anos, principalmente para essa população. Esse aumento está, possivelmente, relacionado com o surgimento de novas medicações, com a ampliação das indicações terapêuticas e também com o crescimento do diagnóstico das doenças depressivas ${ }^{(6-10)}$. Apesar dos resultados positivos, é comum a dificuldade de adesão aos antidepressivos, devido ao tempo de latência para iniciar os efeitos 
terapêuticos e aos efeitos colaterais presentes. A partir desse tema, alguns estudos trazem a importância do papel do profissional da saúde na orientação do usuário do medicamento, a fim de estabelecer um tratamento seguro e adequado ${ }^{(11)}$. De acordo com a Organização Mundial da Saúde, o estilo de vida se define como o conjunto de hábitos e costumes que são influenciados, modificados, encorajados ou inibidos pelo prolongado processo de socialização. Esses hábitos e costumes incluem o uso de substâncias como o álcool, fumo, chá ou café, hábitos dietéticos e de exercício. Eles possuem grande impacto na saúde, com estudos recentes mostrando que mudanças no estilo de vida têm grande impacto na qualidade de vida, a qual envolve o bemestar físico, mental, psicológico e emocional ${ }^{(12)}$.No intuito de avaliar o estilo de vida dos pacientes, foi encontrado na literatura o questionário FANTASTICO, desenvolvido no Canadá que aborda os aspectos físicos, psicológicos e sociais ${ }^{(13)}$. Sendo assim, o objetivo deste trabalho foi avaliar o estilo de vida a partir do instrumento FANTASTICO e identificar o uso de medicamentos antidepressivos associados a determinados comportamentos, em estudantes de medicina.

\section{MÉTODOS}

Trata-se de um estudo de caráter descritivo e transversal, de base populacional, que foi realizado com a participação dos estudantes do curso de medicina em uma Faculdade de Medicina privada, denominada Universidade de Franca (UNIFRAN), em uma cidade no interior do Estado de São Paulo, durante os anos de 2020 e 2021. A coleta dos dados foi realizada por meio de um formulário eletrônicoelaborado no Google Formsonline, onde os participantes responderam via redes sociais virtuais e WHATSAPP, com compartilhamentos do link. Aplicou-se um questionário sócio demográfico, composto por questões referentes a iniciais do nome, sexo, idade, período que está matriculado no curso, aspectos religiosos, uso de medicação para ansiedade e depressão, tempo de uso, como tem acesso a medicações, classe farmacológica, fatores precipitantes e sobre a consciência dos riscos. Este instrumento foi criado a fim de avaliar o uso descontrolado e da aquisição de forma ilegal de medicamentos, que com utilização inadequada pode causar diversos efeitos colaterais, dependência e dificuldades para o desmame da substância, que se tornou comum no meio universitário onde ocorre um aumento progressivo do uso. Para avaliar o estilo de vida, aplicou-se o instrumento FANTASTICO ${ }^{(13)}$, que possui 25 questões fechadas que exploram nove domínios sobre os componentes físicos, psicológicos e sociais do estilo de vida, e apresenta no total 30 questões (itens), todas elas com resposta fechada, que exploram dez domínios dos componentes físicos, psicológicos e sociais do estilo de vida e que se identificam com a sigla "FANTÁSTICO" (ANEXO 1): F - Família e Amigos; A Atividade física/ Associativismo; N - Nutrição; T - Tabaco; A Álcool e Outras drogas; S - Sono/ Stress; T - Trabalho/Tipo de personalidade; I - Introspecção; C - Comportamentos de saúde e sexual; O - Outros Comportamentos. Os itens têm três opções de resposta com um valor numérico de 0,1 ou 2 .

As alternativas estão dispostas em três linhas para facilitar a sua codificação, e a primeira alternativa ( $1^{\mathrm{a}}$ linha) é sempre a de maior valor ou de maior relação com um estilo de vida saudável. A codificação das questões é realizada da seguinte maneira: 2 para a $1^{\text {a }}$ linha, 1 para a $2^{\mathrm{a}}$ linha e zero para a $3^{\mathrm{a}}$ linha, somando-se estes valores em cada domínio e multiplicando-os por dois, obtemos o valor correspondente para cada domínio. A soma de todos os pontos resultantes de todos os domínios permite chegar a um score global que classifica os indivíduos de 0 a 120 pontos. O Guia para universidades saudáveis e outras instituições de educação superior propõem cinco níveis de classificação que estratificam o comportamento em: de 0 a 46 (Necessita melhorar); de 47 a 72 (Regular); de 73 a 84 (Bom); de 85 a 102 (Muito bom) e de 103 a 120 (Excelente). É desejável que os indivíduos atinjam a classificação "Bom". Quanto menor o escore, maior a necessidade de mudança. De maneira geral os resultados podem ser interpretados da seguinte maneira: "Excelente" indica que o estilo de vida proporciona ótima influência para a saúde; "Muito bom" indica que o estilo de vida proporciona considerável influência para a saúde; "Bom" indica que o estilo de vida proporciona muita influência para a saúde; "Regular" indica que o estilo de vida proporciona alguma influência para a saúde, porém apresenta também riscos; "Necessita melhorar" indica que estilo de vida apresenta muitos fatores de risco.

Todas as informações obtidas durante a coleta de dados, foram digitadas duplamente em Banco de Dados no Software Microsoft Excel e passaram por um processo de categorização de acordo com as características das variáveis. Em seguida os dados foram transferidos para o programa estatístico STATA 9.0 para cálculos de frequência absoluta e relativa. Para a síntese das informações e a caracterização da amostra foi utilizada a estatística descritiva, com medidas de tendência central (média e mediana), variabilidade (desvio padrão) e dispersão (mínimo e máximo). As variáveis qualitativas foram descritas por meio de média e desvio padrão. Para medir o grau de associação entre as duas variáveis, utilizou-se o Coeficiente de Correlação de Spearman. Como classificação do grau de correlação, ou seja, da força entre as variáveis, utilizou-se os seguintes parâmetros: positiva ínfima quando $\rho=0,01$ a 0,09 ; positiva baixa quando $\rho=0,10$ a 0,29 ; positiva moderada quando $\rho=0,30$ a 0,49 ; $0,00=$ nenhuma correlação; negativa ínfima quando $\rho=-0,01$ a 0,09 ; negativa baixa quando $\rho=-0,10$ a 0,29 ; negativa moderada quando $\rho$ $=0,30$ a 0,49 . Para a realização desse estudo, foram respeitados os preceitos éticos, tendo sido aprovado pelo Comitê de Ética em Pesquisa da Universidade de Franca, SP, CAAE $\mathrm{n}^{\circ} 32154820.8 .0000 .5495$.

\section{RESULTADOS}

Participaram do estudo 105 estudantes regularmente matriculados no curso de medicina, composto por $82(86,1 \%)$ mulheres e $23(24,1 \%)$ homens com idade média de $22,62 \pm 2,81$ anos, nas respectivas etapas cursadas: $1^{\circ}$ ano: $19(19,9 \%), 2^{\circ}$ ano: $23(24,1 \%), 3^{\circ}$ ano: $16(16,8 \%)$ e $4^{\circ}$ ano $47(49,3 \%)$. Em relação aos aspectos pessoais relacionados à religiosidade, $82(86,1 \%)$ dos alunos afirmaram ser religiosos e a frequência de participação em atividades religiosas seguem os seguintes dados: algumas vezes: 58 (60,9\%); mensalmente: $9(9,45 \%)$ e semanalmente: 38 (39,9\%). A Tabela 1 apresenta a distribuição das respostas, referente aos aspectos relacionados ao uso de fármacos $\mathrm{e}$ seus fatores precipitantes. Com o objetivo de avaliar o estilo de vida dos estudantes de medicina, as respostas associadas ao questionário FANTÁSTICO, foram analisadas de acordo com cada domínio presente na escala. A Tabela 2 apresenta a distribuição das respostas dos domínios presentes na escala. Os resultados do questionário revelaram um valor médio global de $64,28 \pm 0,73$ pontos, que se enquadra na categoria de 55 a 69 pontos, ou seja, classificação de um estilo de vida considerado "Bom". A distribuição do escore de acordo com os valores médios estão apresentados na Tabela 3. No presente estudo, nenhum participante apresentou pontuação na categoria "Necessita Melhorar", no entanto, apenas 2 (1,9\%) se enquadraram na categoria "Excelente", classificação máxima. A correlação dos aspectos apresentados relacionados aos domínios do questionário "Estilo de vida fantástico" com o uso de antidepressivos, bem como a frequência de participação das atividades religiosas estão apresentados na Tabela 4.

\section{DISCUSSÃO}

De acordo com os resultados acima citados, os domínios que mais necessitam de mudança nos estudantes de medicina, estão relacionados principalmente ao "Tipo de Comportamento" (1,3 pontos), "Nutrição" (1,5 pontos) e "Atividade" (1,6 pontos), dentro desses aspectos, os estudantes demonstram sempre estar com pressa, frequentemente comem em excesso açúcar, sal, gordura animal e alimentos industrializados, além de não serem vigorosamente ativos pelo menos durante 30 minutos por dia com a associação de corrida, bicicleta, dentre outras atividades, considerando a pontuação máxima de 4 pontos para cada item avaliado. 
Tabela 1. Distribuição das respostas sobre o uso de medicamentos antidepressivos de todos os estudantes. Franca, 2021.

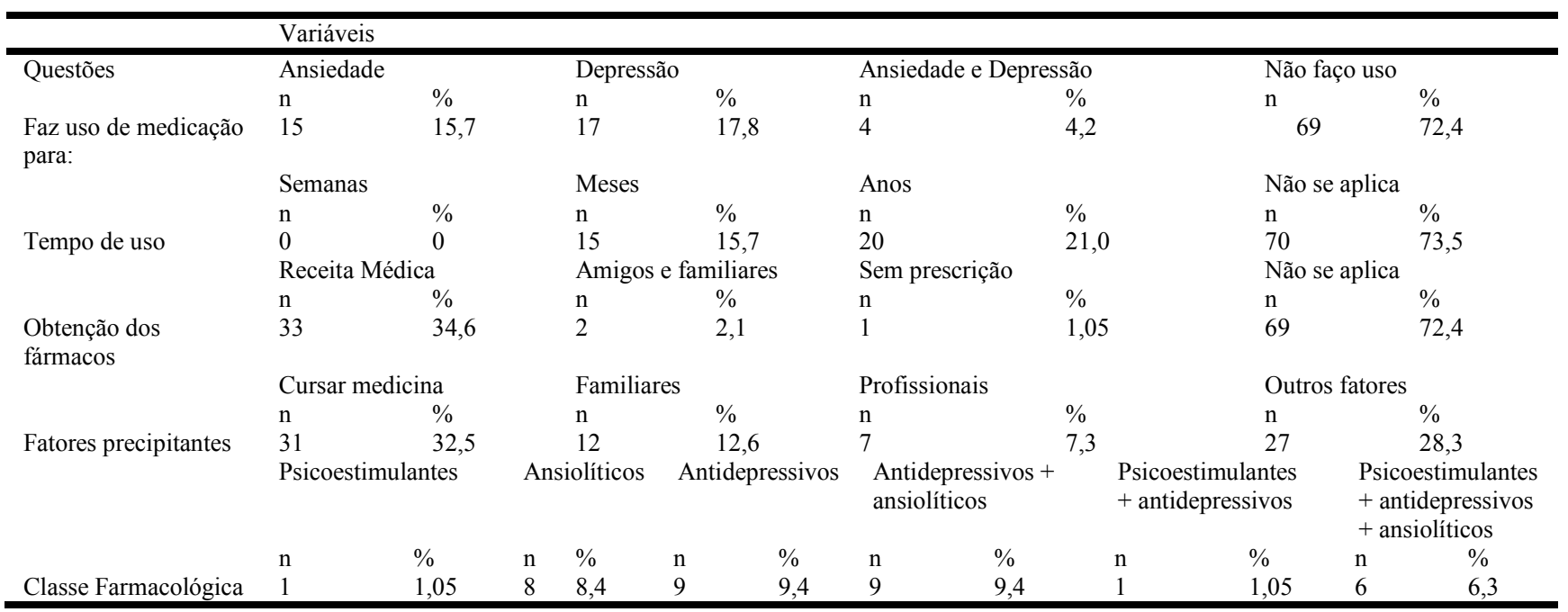

Tabela 2. Distribuição das respostas dos domínios do questionário "Estilo de vida fantástico" em números relativos e absolutos, apresentada em média/ desvio padrão de todos os estudantes de medicina. Franca, 2021

\begin{tabular}{|c|c|c|c|c|c|c|c|c|c|c|c|}
\hline & Scores & & & & & & & & & & \\
\hline & 0 & & 1 & & 2 & & 3 & & 4 & & \\
\hline Itens da Escala & $\mathrm{n}$ & $\%$ & $\mathrm{n}$ & $\%$ & $\mathrm{n}$ & $\%$ & $\mathrm{n}$ & $\%$ & $\mathrm{n}$ & $\%$ & Média $\pm \mathrm{DP}$ \\
\hline Família e Amigos & 1 & 0,95 & 1 & 0,95 & 10 & 9,5 & 19 & 18,1 & 74 & 70,4 & $3,42 \pm 0,83$ \\
\hline \multirow[t]{2}{*}{ Atividade } & 40 & 38 & 16 & 15,2 & 15 & 14,2 & 19 & 18,1 & 15 & 14,2 & $1,61 \pm 1,46$ \\
\hline & 27 & 25,7 & 29 & 27,6 & 17 & 16,1 & 15 & 14,2 & 17 & 16,1 & \\
\hline \multirow[t]{2}{*}{ Nutrição } & 0 & 0 & 12 & 11,4 & 40 & 38,1 & 29 & 27,6 & 16 & 15,2 & $2,36 \pm 1,25$ \\
\hline & 12 & 11,4 & 9 & 8,5 & 22 & 20,9 & 19 & 18,1 & 43 & 40,9 & \\
\hline \multirow[t]{4}{*}{ Cigarro e Drogas } & 1 & 0,95 & 3 & 2,8 & 22 & 20,9 & 4 & 3,8 & 75 & 71,4 & $3,29 \pm 1,10$ \\
\hline & 18 & 17,1 & 0 & 0,0 & 0 & 0,0 & 0 & 0 & 87 & 82,8 & \\
\hline & 0 & 0 & 2 & 1,9 & 8 & 7,6 & 13 & 12,3 & 82 & 78,1 & \\
\hline & 4 & 3,8 & 2 & 1,9 & 23 & 21,9 & 63 & 60,0 & 13 & 12,3 & \\
\hline Álcool & 0 & 0 & 2 & 1,9 & 7 & 6,6 & 15 & 14,2 & 79 & 75,2 & $2,78 \pm 1,38$ \\
\hline \multirow[t]{3}{*}{ sexo seguro } & 4 & 3,8 & 13 & 12,3 & 37 & 35,2 & 44 & 41,9 & 7 & 6,67 & \\
\hline & 0 & 0 & 9 & 8,5 & 37 & 35,2 & 41 & 39,0 & 17 & 16,1 & \\
\hline & 11 & 10,4 & 0 & 0,0 & 18 & 17,1 & 26 & 24,7 & 50 & 47,6 & \\
\hline \multirow[t]{2}{*}{ Tipo de comportamento } & 32 & 30,4 & 27 & 25,7 & 34 & 32,3 & 9 & 8,5 & 3 & 2,86 & $1,65 \pm 1,11$ \\
\hline & 11 & 10,4 & 13 & 12,3 & 49 & 46,6 & 26 & 24,7 & 6 & 5,71 & \\
\hline \multirow[t]{3}{*}{ Introspecção } & 10 & 9,5 & 9 & 8,5 & 35 & 33,3 & 32 & 30,4 & 19 & 18,1 & $2,01 \pm 1,10$ \\
\hline & 16 & 15,2 & 23 & 21,9 & 49 & 46,6 & 12 & 11,4 & 5 & 4,76 & \\
\hline & 11 & 10,4 & 14 & 13,3 & 54 & 51,4 & 20 & 19,0 & 6 & 5,71 & \\
\hline Trabalho & 5 & 4,7 & 8 & 7,6 & 29 & 27,6 & 45 & 42,8 & 18 & 17,1 & $2,60 \pm 1,02$ \\
\hline
\end{tabular}

Tabela 3. Distribuição da frequência dos valores do escore total do questionário "Estilo de vida fantástico", dos estudantes de medicina. Franca, 2021

\begin{tabular}{lllll}
\hline Mínimo & Máximo & Mediana & Média & Desvio Padrão \\
\hline 42 & 86 & 65 & 64,28 & 0,73 \\
\hline
\end{tabular}

Tabela 4. Correlação entre o estilo de vida e uso de antidepressivos e frequência de participação das atividades religiosas dos estudantes de medicina. Franca, 2021

\begin{tabular}{lll}
\hline Itens da Escala & $\begin{array}{l}\text { Uso de antidepressivos } \\
(\text { rho }) \text { Spearman }(\rho)\end{array}$ & $\begin{array}{l}\text { Frequência de Participação das atividades religiosas } \\
\text { (rho) Spearman }(\rho)\end{array}$ \\
\hline Família e Amigos & $-0,01$ & 0,06 \\
Atividade & 0,06 & $-0,03$ \\
Nutrição & 0,14 & 0,21 \\
Cigarro e Drogas & 0,12 & 0,26 \\
Álcool & 0,06 & 0,19 \\
Sono, cinto de segurança, estresse e sexo seguro & 0,24 & 0,10 \\
Tipo de comportamento & 0,09 & $-0,09$ \\
Introspecção & 0,20 & 0,05 \\
Trabalho & 0,03 & 0,01 \\
\hline Coeficiente de Correlação de Spearman $(\rho)$. &
\end{tabular}


Em relação aos aspectos melhores pontuados, no domínio "Cigarro e Drogas", os estudantes de medicina obtiveram práticas saudáveis nessa dimensão, já que a maioria deles referiram não abusarem de remédios ou drogas em exagero; no domínio "Família e Amigos", a maioria dos estudantes relatam que tem com quem falar das coisas que são importantes para eles, na mesma proporção que oferecem e recebem afeto; no domínio "Álcool”, 79 (75,2\%) dos estudantes ingerem quantidades mínimas apresentadas, ou seja de 0 a 7 doses alcoólicas semanais e apenas $2(1,9 \%)$ estudantes ingerem mais de 20 doses por semana; e $94(89,5 \%)$ dos estudantes usam cinto de segurança a maioria das vezes ou sempre, assim como no mesmo domínio $76(72,3 \%)$ praticam sexo seguro com relativa frequência ou sempre; $62 \quad(59,0 \%)$ consideram não dormirem bem $\mathrm{e}$ consequentemente estarem descansados, e cerca de metade dos estudantes $54(51,4 \%)$ não são capazes de lidar com o estresse do diaa-dia. Os aspectos relacionados ao domínio "Introspecção" mostraram que $88(83,8 \%)$ dos estudantes avaliados sentem-se tensos e desapontados, tristes e deprimidos 79 (75,2\%), mas buscam com relativa frequência manterem pensamentos otimistas $51(48,5 \%)$. Entretanto, o domínio "Trabalho" demonstrou que 63 (60\%) dos estudantes estão satisfeitos com seus trabalhos e/ou funções. $\mathrm{O}$ estudo permitiu identificar que $34,2 \%$ dos estudantes referiram o uso de algum medicamento, com maior índice de tempo iniciado a mais de um ano, direcionado principalmente ao tratamento da Depressão 17 $(17,8 \%)$ e com a prescrição individualizada de receita médica. O principal fator precipitante relacionado ao uso de medicamentos antidepressivos, esteve associado ao fato de cursar medicina; e de acordo com a classe farmacológica, os antidepressivos e os ansiolíticos foram os mais relatados dentre os estudantes.

A análise dos dados apresentados, mostrou que, o uso de antidepressivos apresentou correlação linear positiva ínfima, com o estilo de vida relacionado aos domínios atividade, álcool, tipo de comportamento e trabalho, e correlação linear positiva baixa com o estilo de vida relacionado aos domínios de nutrição, cigarro e drogas, sono, cinto de segurança, estresse e sexo seguro e introspecção, ou seja, há uma correlação diretamente proporcional com a baixa classificação do estilo de vida associada ao uso de antidepressivos; com exceção ao domínio "Família e Amigos" que apresentou correlação linear negativa baixa ao uso de antidepressivos, ou seja, correlação inversamente proporcional aos aspectos avaliados. $\mathrm{Na}$ análise entre a frequência de participação das atividades religiosas com o estilo de vida, houve correlação linear positiva ínfima entre os domínios: família e amigos, introspecção e trabalho e correlação linear positiva baixa com os domínios: nutrição, cigarro e drogas, álcool, sono, cinto de segurança, estresse e sexo seguro, ou seja, quanto maior a frequência de participação das atividades religiosas, maior o escore resultante dos itens avaliados pelo questionário "Estilo de vida fantástico". A frequência de participação das atividades religiosas, não apresentou influência sobre os domínios "atividade" e "tipo de comportamento", pois houve correlação negativa ínfima entre os aspectos avaliados, evidenciando na presente amostra, uma correlação inversamente proporcional nestes últimos aspectos. Com base nos domínios "hábitos e vícios" abordados e avaliados no decorrer do estudo, de um modo geral identificou-se que dentre os estudantes de medicina, a grande maioria enquadra-se como classificação geral "bom". Em comparação com estudo de Tassini CC et al, avaliando estudantes de medicina e fisioterapia demonstrou um quadro inferior, sendo $79,6 \%$ "Regular", apresentando $1,6 \%$ "Necessita Melhorar", evidenciando grande diferença nesse aspecto (14). Camargo e Bueno declaram que muitas vezes, há um problema em conciliar as necessidades fisiológicas e psicológicas com as exigências do trabalho e que deste conflito, pode desencadear repercussões sobre a saúde física e mental. Da mesma forma, nosso estudo mostra que a maioria dos participantes refere não dormir bem $(59 \%)$ e não serem capazes de lidar com o estresse do dia-a-dia $(51,4 \%)^{(15)}$. A faculdade implica para muitos, deixar a casa dos pais, alterando fortemente os hábitos alimentares. Nessa fase estão mais preocupados com o bom desempenho acadêmico e deixam de lado a importância de uma alimentação saudável, procurando alternativas com baixo aporte nutricional ${ }^{(16)}$. Na presente investigação, os domínios "Nutrição" (1,5 pontos) e "Atividade" (1,6 pontos), são os que mais necessitam de mudança, apresentando indivíduos pouco saudáveis. Dessa forma, como descrevem Silva et al, existe a possibilidade de orientar os universitários quanto à importância do estilo de vida saudável e incentivar programas de atividade física na universidade ${ }^{(17)}$. Um estudo realizado em estudantes de medicina de uma Universidade privada do Paraná utilizando o Questionário Fantástico, evidenciou que os participantes possuem uma má qualidade de vida relacionada com uso de cigarro, drogas e álcool para ambos os sexos, enquanto o presente estudo, também relacionado aos estudantes de medicina, porém de uma Universidade particular de São Paulo, demonstrou deficiência nos domínios "tipo de comportamento", "atividade física" e "nutrição" ${ }^{(18)}$.

\section{CONCLUSÃO}

Com base nos resultados do presente estudo, sugere-se que o uso de antidepressivos, foi fator preditor para uma indicação de Estilo de Vida não saudável de acordo com o questionário estilo de vida FANTÁSTICO.

\section{REFERENCIAS}

Añez CRR, Reis RS, Petroski EL. Versão brasileira do questionário "Estilo de vida FANTÁSTICO": tradução e validação para adultos jovens. Arquivos Brasileiros de Cardiologia. 2008 Agosto; 91.

Camargo RA, Bueno SM. Leisure, life besides work for a soccer team of hospital workers. Rev Latino Am Enfermagem. 2003; 11(4):490-8

Carvalho A. Depressão e outras perturbações mentais comuns. 2017.

Clemente AS. Concepções dos psiquiatras sobre o transtorno bipolar do humor e sobre o estigma a ele associado. 2015.

Feitosa EPS. et al. Hábitos alimentares de estudantes de uma Universidade pública no Nordeste, Brasil. Alim. Nutr. Araraquara, v. 21, n. 2, p. 225-230,2010.

Finley P. R.; Laird L. K.; Benefield W. H. Mood disorders I: major depressive disorders. In: KODA-KIMBLE, M. A. et al. Applied therapeutics: the clinical use of drugs. Philadelphia: Lippincott Williams \& Wilkins, 2004.

Garcias CMM, Pinheiro RT, Garcias GDL, Horta BL, Brum CB. Prevalência e fatores associado ao uso de antidepressivos em adultos de área urbana de Pelotas, Rio Grande do Sul, Brasil, em 2006. Cad Saúde Pública 2008; 24(7):1565-1571.

Luna ISD, Augusta A, Ferrari F, Allan Lobo da Costa AP, Silva Gd. Consumo de psicofármacos entre alunos de medicina do primeiro e sexto ano de uma universidade do estado de São Paulo. 2018 jan-abr; 10.

Oliveira Natan Nascimento, et al.. Estilo de vida de estudantes universitários. Maringá; 2019.

Portes LA. Estilo de Vida e Qualidade de Vida: semelhanças e diferenças entre os conceitos.

Ribeiro AG, Cruz LPd, Marchi KC, Tirapelli CR, Miasso AI. Antidepressivos: uso, adesão e conhecimento entre estudantes de medicina. Ciência \& Saúde Coletiva. 2014; 19.

Sadock BJ, Sadock VA. Compêndio de psiquiatria: ciência do comportamento e psiquiatria clínica. 9 ${ }^{\mathrm{a}}$ ed. Porto Alegre: Artemd; 2007.

Schmitt R, Gazalle FK, Lima MS, Cunha A, Souza J, Kapczinski F. The efficacy of antidepressants for generalized anxiety disorder: a systematic review and meta-analysis. Rev Bras Psiquiatr 2005; 27(1):18-24.

Scolaro LL, Bastiani Dd, Mella EAC. Avaliação do uso de antidepressivos por estudantes de uma instituição de ensino superior. UNIPAR. 2010 set./dez.; 14.

Silva DA, Quadros TM, Gordia AP, Petroski EL. Association of overweight with socio-demographic variables and lifestyle among Brazilian university students. Ciên Saúde Colet. 2011;16(11):4473-9.http://dx.doi.org/10.1590/S141381232011001200020 
Tassini CC; Val GR; Candido SS; Bachur CK. Avaliação do Estilo de Vida em Discentes Universitários da Área da Saúde através do Questionário Fantástico. Int. J. Cardiovasc. Sci. [online]. 2017, vol.30, n.2 [cited 2021-05-04],pp.117-122.

Teixeira PR, Paiva V, Shimma E. Ta difícil de engolir? São Paulo (SP): Nepaids; 2000.
Usala T, Clavenna A, Zuddas A, Bonati M. Randomised controlled trial of selective serotonin reuptake inhibitors in treating depression in children and adolescents: a systematic review and meta-analysis. Eur Neuropsychopharmacol 2008; 18(1):62-73. 